\title{
A System-Wide Population Health Value Approach to Reduce Hospitalization Among Chronic Kidney Disease Patients: an Observational Study
}

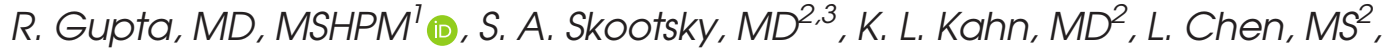 \\ F. Abtin, MD', S. Kee, MD', S. B. Nicholas, MD, MPH, $P h D^{5}$, S. Vangala, $M S^{2}$, and J. Wilson, \\ $M D^{5}$
}

'Department of Internal Medicine, UCD Health, Sacramento, CA, USA; ${ }^{2}$ Los Angeles, USA; ${ }^{3}$ UCLA Department of Medicine, Division of General Internal Medicine and Health Services Research, Los Angeles, CA, USA; ${ }^{4}$ UCLA Health, Los Angeles, CA, USA; ${ }^{4}$ CLA Department of Radiology, Los Angeles, CA, USA.

BACKGROUND: Chronic kidney disease (CKD) is a leading cause of healthcare morbidity, utilization, and expenditures nationally, and caring for late-stage CKD populations is complex. Improving health system efficiency could mitigate these outcomes and, in the COVID-19 era, reduce risks of viral exposure.

OBJECTIVE: As part of a system-wide transformation to improve healthcare value among populations with high healthcare utilization and morbidity, UCLA Health evaluated a new patient-centered approach that we hypothesized would reduce inpatient utilization for CKD patients. DESIGN: For 18 months in 2015-2016 and 12 months in 2017, we conducted an interrupted time series regression analysis to evaluate the intervention's impact on inpatient utilization. We used internal electronic health records and claims data across six payers.

PARTICIPANTS: A total of 1442 stage 4-5 CKD patients at a large academic medical center.

INTERVENTION: Between October and December 2016, the organization implemented a Population Health Value CKD intervention for the CKD stages $4-5$ population. A multispecialty leadership team risk stratified the population and identified improvement opportunities, redesigned multispecialty care coordination pathways across settings, and developed greater ambulatory infrastructure to support care needs.

MAIN MEASURES: Outcomes included utilization of hospitalizations, emergency department (ED) visits, inpatient bed days, and 30-day all-cause readmissions.

KEY RESULTS: During the 12 months following intervention implementation, the monthly estimated rate of decline for hospitalizations was 5.4\% (95\% CI: 3.4-7.4\%), which was 3.4 percentage points faster than the 18month pre-intervention decline of $2.0 \%$ (95\% CI: 1.0 $2.2 \%)$ per month $(p=0.004)$. Medicare CKD patients' monthly ED visit rate of decline was 3.0\% (95\% CI: $1.2-$ $4.8 \%$ ) after intervention, which was 2.6 percentage points

Electronic supplementary material The online version of this article (https://doi.org/10.1007/s11606-020-06272-5) contains supplementary material, which is available to authorized users.

Received May 30, 2020

Accepted September 25, 2020

Published online November 2, 2020 faster than the pre-intervention decline of $0.4 \%$ (95\% CI: -0.8 to $1.6 \%$ ) per month ( $p=0.02$ ).

CONCLUSIONS: By creating care pathways that link primary and specialty care teams across settings with increased ambulatory infrastructure, healthcare systems have potential to reduce inpatient healthcare utilization.

J Gen Intern Med 36(6):1613-21

DOI: $10.1007 / \mathrm{s} 11606-020-06272-5$

(c) Society of General Internal Medicine 2020

\section{INTRODUCTION}

There is a national imperative to provide coordinated, highquality healthcare for chronic conditions out of the hospital to reduce the public's COVID-19 exposure risk, utilization, and spending. ${ }^{1-3}$ Commercial and public payers are increasingly incentivizing proactive disease management for current and future high-risk, high-cost patients such as those with chronic kidney disease (CKD). ${ }^{4-7}$ For example, in 2019, the Center for Medicare and Medicaid Innovation proposed payment models to enhance caring for CKD patients. 8,9

Care for CKD is a leading cause of annual spending nationally ( $\$ 114$ billion). Among patients with CKD stage 4 and CKD stage 5 (CKD 4-5), healthcare utilization across all payers is eight times higher than for the general population with an average annual all-cause spending per patient of $\$ 77,000$ (commercial payers) and $\$ 46,100$ (Medicare). ${ }^{10-15}$

As kidney disease stage advances, so does the incidence of concurrent conditions, end organ damage, complex ambulatory management, specialty care, and hospitalization risk. ${ }^{16-18}$ Surprisingly, proactive care coordination efforts and planning for dialysis or transplant have not systematically kept pace with these documented needs. ${ }^{18,19}$ This lack of coordination can lead to increases of hemodialysis catheter malfunction, hospitalizations in the first months of dialysis, mortality, and costs. ${ }^{17,} 20,21$

In recognition of the complex and accelerating needs of high-risk, high-cost patients, UCLA Health developed its Population Health Value (PHV) approach. This program has guided the healthcare system to apply improvement 
opportunities to its CKD population. This study documents and evaluates the system-wide intervention with the goal of reducing hospitalizations.

\section{METHODS}

\section{Context}

Several empirical analyses at UCLA identified CKD 4-5 patients as a sub-cohort with high morbidity, costs, and potential to benefit from focused interventions. For example, consistent with the literature, ${ }^{22}$ our findings among these patients revealed frequent hospitalizations associated with an emergent need to initiate dialysis and treat malfunctioning catheters. ${ }^{13,23,24}$ Additionally, consistent with national findings, ${ }^{25} 53 \%$ of CKD $4-5$ patients had never had a documented nephrology evaluation, potentially missing proactive care. Given the importance of CKD stage in predicting these outcomes, the institution focused its initial PHV-CKD interventions on its attributed (Appendix-Section I) CKD 4-5 patients.

UCLA created a multispecialty leadership team who used actionable electronic health records and claims data to risk stratify and identify improvement opportunities for the entire institution's population of CKD 4-5. The primary intervention components included redesigning proactive care coordination pathways that bridge primary and multispecialty care through a system-wide CKD care team $^{26-33}$ and developing greater ambulatory infrastructure to support care needs. ${ }^{34,35}$

\section{Study Design}

We used a multiple time series design evaluating outcomes during the pre-intervention (April 1, 2015-September 30,2016 ) and post-intervention (January 1, 2017-December 31, 2017) periods, excluding the implementation period (October 1, 2016-December 31, 2016) (Fig. 1).

\section{Setting}

UCLA Health is associated with the David Geffen School of Medicine that encompasses four hospitals, 150 ambulatory clinics, and 1470 clinical faculty. In 2015, the institution participated in six accountable care (ACO) contracts and underwent primary care expansion nearly doubling the patient network. $^{23}$ To enhance care for patients, UCLA had previously introduced primary care coordinators to facilitate patients with post-hospitalization, behavioral health, and preventive health needs. ${ }^{33,36}$ Primary care coordinators, however, recognized that they needed more time to build relationships with CKD specialty care teams (i.e., nephrology, interventional radiology) and to keep current with specialty workflows and resources. These time-consuming activities were not feasible when any single coordinator's patient panel included only a few CKD 4-5 patients. In response to these experiences, the idea of consolidating advanced CKD patients to a dedicated CKD care coordinator emerged to facilitate reliable, rapid care coordination for CKD patients with declining kidney function. $^{23}$

\section{Data Sources and Measures}

CKD patient stage and clinical characteristics were defined using EPIC@ electronic health records (EHR) and health plan claims data across six payers at the time of patient study entry. Patient attribution and utilization outcomes data capturing both local EHR and claims encounters were collected over the full study period (Appendix - Section I, metric definitions and validation). We analyzed four available hospital-based outcomes that were collected concurrently and were assessed to be valid and complete. These included hospitalizations, ED visits, inpatient bed days, and 30-day all-cause readmissions per 1000 patient-years.

The analysis cohort included two mutually exclusive patient groups with a diagnosis of CKD 4-5. Original entrants were attributed as of April 1, 2014; new entrants were attributed between April 2, 2014, and September 30, 2016, when UCLA Health primary care was expanded by nearly 22 practices. New entrants were demographically younger, had fewer concurrent conditions, and had a lower proportion of high-risk patients (Appendix - Section II, exhibit B). Both the original entrant and new entrant patients received the intervention unless they met exclusion criteria, which included receiving a kidney transplant, exiting prior to exposure to the PHV-CKD intervention, no longer meeting the rules of the attribution model or dying prior to September 30, 2016. Exited patients were excluded from post-intervention analyses following their exit date.

Development of the PHV-CKD Intervention. Between August and September 2016, a 15-member multidisciplinary, multispecialty PHV-CKD Leadership Team was developed. Their task was to identify the prevalent needs of the CKD 4-5 population, create trackable metrics, and co-create systemwide interventions to avoid emergent hospitalizations. ${ }^{37}$ Care coordinators had conversations with patients to incorporate patient input.

To develop an aligned system-wide approach, UCLA housed the intervention in a centralized Office of Population Health and Accountable Care, rather than within a single clinical division. This approach supported a system-level commitment to improve care for all CKD 4-5 patients, regardless of having a pre-existing relationship with a nephrologist. To achieve these goals, the Leadership Team defined and developed three support teams: a system-wide CKD Care Team, newly structured Care Coordination Team led by a CKD Care Coordinator (CKD-CC), and PHV Data Team who developed customized measures using claims and EPICC clinical data (Fig. 2). ${ }^{38,39}$ 

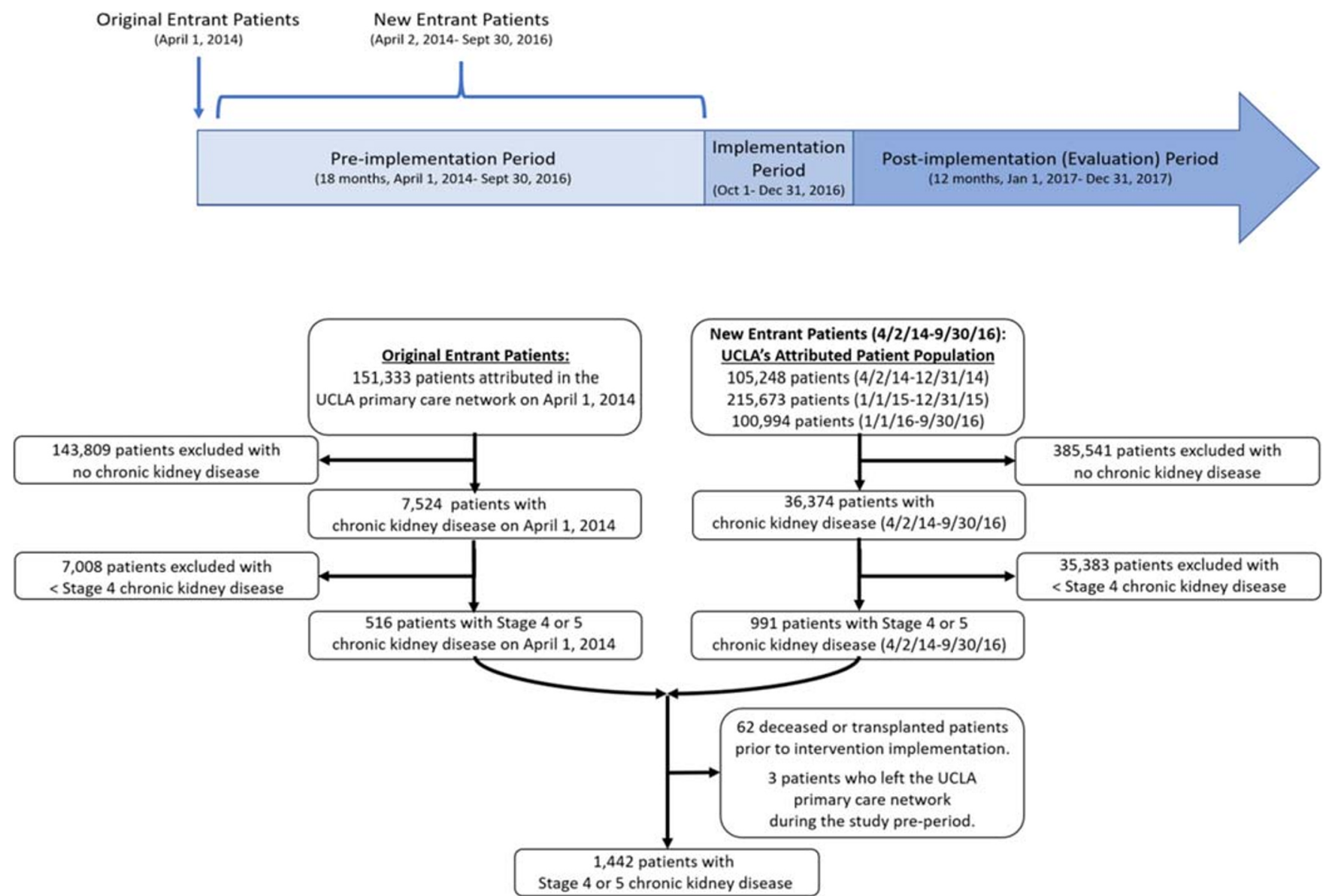

Figure 1 Timeline and flowchart of study participants with chronic kidney disease (CKD). Legend: CKD (UCLA definition) is applied to patients with any of the following: an estimated glomerular filtration rate (eGFR) $<60 \mathrm{~mL} / \mathrm{min} / 1.73 \mathrm{~m}^{2}$ within two previous years (outpatient labs) with no eGFR $\geq 60 \mathrm{~mL} / \mathrm{min} / 1.73 \mathrm{~m}^{2}$ in the last two months; medical chart documentation of inpatient or outpatient hemodialysis; or CKD included in an inpatient problem list, inpatient discharge diagnosis, or outpatient encounter diagnosis. Original entrants were attributed as of April 1, 2014; new entrants were attributed between April 2, 2014, and September 30, 2016, when UCLA Health primary care expanded by nearly 22 practices who were demographically younger, had fewer concurrent conditions, and had a lower proportion of high-risk patients (Appendix-Section II, exhibit B). Both the original entrant and new entrant patients received the intervention unless they met exclusion criteria including receiving a kidney transplant, exiting prior to exposure to the PHV-CKD intervention, no longer meeting the rules of the attribution model or dying prior to September 30, 2016.

Implementing the PHV-CKD Intervention. The three teams identified markers for clinical decline or need (e.g., dialysis access malfunction) and developed a CKD patient registry who could benefit from early intervention. ${ }^{23}$ These patients included those with recent or frequent ED visits, hospitalizations, missed clinic or dialysis visits, recent declines in renal function or weight changes, or new reports of escalating problem(s) from the system-wide CKD Care Team members.

The CKD-CC (one full-time equivalent (FTE) staffed by two coordinators) used standardized care pathways that prioritized early identification of clinical decline or need, avoided duplicate care across siloed specialties, and coordinated proactive strategies to direct patients to the most appropriate venue. ${ }^{38}$ Care coordinators, aware that patients found fleeting contacts with members of siloed clinical settings frustrating, helped patients to engage longitudinally with a dedicated member of the system-wide CKD Care Team to best address their problems.
To execute the care pathways, the CKD-CC worked closely with 40 unlicensed primary care coordinators (PC-CCs) who had historically focused on addressing healthcare maintenance and post-discharge follow-up, however, had limited bandwidth to coordinate care across multiple siloed specialists for CKD 4-5 patients. ${ }^{33}$ By building upon their experience, the CKD-CC with PC-CCs considered the urgency of patient needs, the availability of caregiver supports, existing professional relationships, and staff bandwidth as they linked patients and services (Fig. 2, staff training details).

Figure 3 highlights four scenarios (A-D) describing prevalent patterns of clinical decline or need among CKD 4-5 patients with varying avenues of presentation and engagement with the health system. The figure displays a coherent model by which proactive care coordination pathways can identify patients with clinical decline; assess patient clinical, social, and care delivery needs; and enhance the likelihood of desired outcomes. 


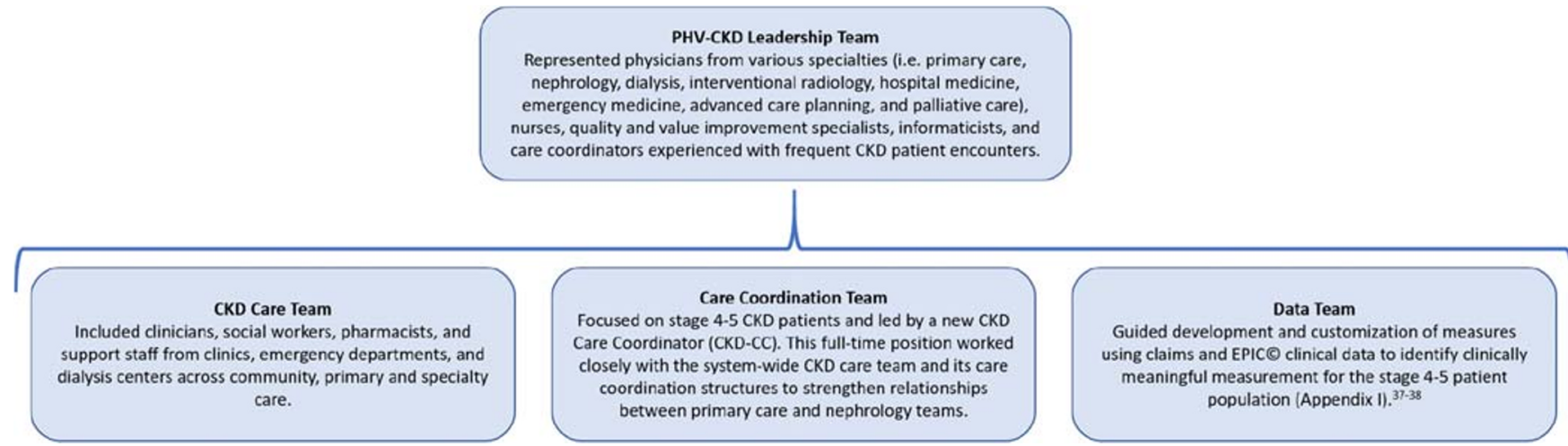

Figure 2 New teams developed to design and implement PHV-CKD intervention. Legend: The health system supported one FTE for the CKD care coordinator. Two primary care coordinators were trained for this role so that they could maintain coverage of their services. The primary care coordinators at UCLA completed nearly $40 \mathrm{~h}$ of initial training under a case manager and social workers. The curriculum included modules about problem solving, patient engagement and motivational interviewing, responsibilities and workflows, post-acute care planning, socio-behavioral risk assessment, physician communication, community resources, and health plan navigation. They also received shadowing experiences with a veteran care coordinator for two weeks, case-based problem solving biweekly, and continuing education through monthly 2-h meetings with other care coordinators to stay current with new programs, policies, and resources across the health system and within the community. ${ }^{33}$ The CKD care coordinator additionally received on-the-job teaching about the nephrology clinic and dialysis center workflows, resources, and contacts. An RN program director, and nurse care manager and licensed clinical social worker were available by telephone to answer complex questions and provide consultation.

The CKD-CC operationalized pathways by communicating with patients and leading care coordination across stakeholders to implement needed interventions. The CKD-CC reviewed the CKD registry iteratively to proactively identify clinical decline or need (Fig. 3, column 1) and then assessed patient needs through phone meetings with the patient and caregivers. She promptly consolidated relevant clinical and social service needs across the system-wide CKD Care Team to align clinical and social resources and define acute care plans (Fig. 3, column 2). The CKD-CC also scheduled nephrology and primary care appointments for goals of care conversations and dialysis initiation (Fig. 3, scenario A, column 2).

If additional clinical needs emerged, the CKD-CC engaged nephrologists and primary care clinicians. The CKD-CC and lead nephrologist were co-located to facilitate this process. If neither the primary consult nephrologist nor the primary care clinician could be reached promptly, the lead nephrologist would develop urgent care plans and determine the timing of needed follow-up. ${ }^{23}$

The CKD-CC also guided appropriate patient care to less costly ambulatory care sites (Fig. 3, column 4) and used motivational interviewing to promote self-management strategies. ${ }^{40}$ This approach substantially differed from routine practice of referring patients with clinical decline or need directly to the ED (Fig. 3, legend). The CKD-CC also monitored intervention fidelity and longitudinal outcomes to improve performance (Fig. 3, column 4).

\section{Developing Greater Ambulatory Infrastructure to Support Care Needs. Care coordination pathways guided patients who could be cared for in ambulatory settings toward primary or specialty care clinics, dialysis centers, or their homes. A network of community resources grew to offer patients early medication management and to address social determinants of}

health. New ambulatory care sites and operation hours emerged so that patients could receive care in the most appropriate, least costly settings. For example, with improved access, ambulatory visits could mitigate hospitalizations when appropriate. ${ }^{34,} 35$ The institution also extended hours at an existing outpatient interventional radiology suite by staggering shifts to address catheterrelated problems with fewer delays.

Based on the focus and structure of this multi-pronged intervention, we hypothesized that the PHV-CKD interventions, which prioritized ambulatory care coordination, would reduce hospitalizations and emergency department utilization among CKD 4-5 patients.

\section{Analysis}

We determined frequencies, means, medians, and IQRs of CKD 4-5 patient demographics overall, stratified by payer type (i.e., Medicare versus commercial) at the time of study entry. Comparisons between strata were performed using $t$ tests for continuous variables, and chi-squared or Fisher's exact tests as appropriate for categorical variables. We used an interrupted time series approach, a strong longitudinal quasi-experimental design to evaluate the impact of the PHV-CKD intervention on utilization outcomes. ${ }^{41,42}$ All outcomes were specified as rates per 1000 patient-years to account for the number of patients in the denominator at each time period. Using negative binomial marginal models, ${ }^{43}$ we examined study outcomes monthly across the study period. Primary model terms included months since the intervention, study period (pre- versus post-intervention), and the interaction of these terms. Regression models controlled for age, gender, race, ethnicity, number of comorbidities, and pre-intervention 


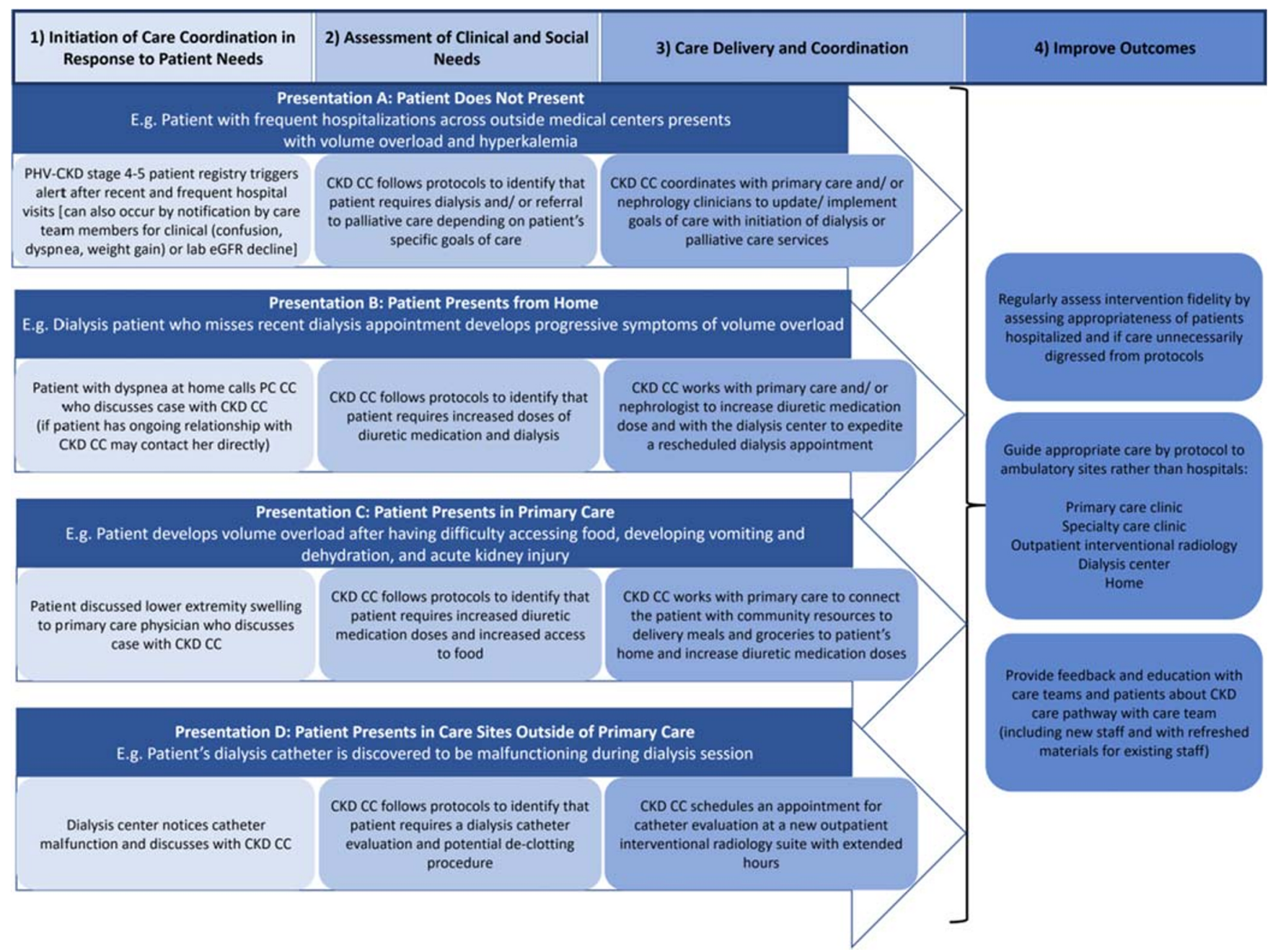

Figure 3 Examples of the application of the PHV-CKD care coordination pathway to four prevalent scenarios (A-D) describing clinical decline or need for chronic kidney disease patients. Legend: Baseline structural problems prior to pathway implementation: Presentation A describes patients without a regular pattern of care monitoring or consistent care clinician, but with frequent episodes of clinical decline or need prompting recurrent ED and hospital stays across external care settings. The system lacked a patient registry for CKD patients to monitor patients outside of in-person clinic visits, data systems to integrate plans data useful in monitoring patients outside of the local hospital and ED, and staff to proactively monitor stage 4-5 CKD patients. Presentation B describes patients with a regular clinician but inconsistent followthrough with visits. From this group, analytics documented the frequent incidence of dialysis patients presenting with shortness of breath after missing a dialysis appointment. Primary care team including primary care coordinators were spread thin and did not have dedicated time to expeditiously care for these patients who require significant coordination outside of in-person visits. Presentation $\mathrm{C}$ focuses on patients with a recent primary care evaluation showing acute on chronic kidney injury. Primary care clinics were not linked to the operations of specialty services required to expeditiously care for patients. Presentation D describes patients who present with urgent or emergent needs from a variety of other care settings with malfunctioning dialysis catheters. The health system lacked staff to coordinate specialty care and care sites to evaluate and treat catheters in the ambulatory setting, and primary and multispecialty care team members would often send patients to the ED for evaluation. The system-wide CKD care team both includes primary care members consisting of primary care coordinators, primary care clinicians, social workers, and others and includes multispecialty care members consisting of specialists (e.g., nephrologists), emergency department, urgent care, dialysis centers, and others. CKD-CC, chronic kidney disease care coordinator; PC-CC, primary care coordinator.

high-risk or high-utilizer patient status. We report estimated monthly rates of change for each outcome in the preintervention and post-intervention periods. We performed a sensitivity analysis to evaluate the models including the intervention period in addition to the pre- and postintervention periods. Since results remained unchanged, we present results comparing trends in outcomes during the pre- and post-intervention periods.

We conducted sensitivity analyses to compare original versus new entrants to the study cohort to assess for bias in changes of the patient population over time. We also assessed effect modification between Medicare and other payer groups, and patients who were CKD stage 4 versus $\mathrm{CKD}$ stage 5 at study entry. We used SAS software, version 9.4, for all analyses.

\section{RESULTS}

We analyzed utilization outcomes during the pre- and postintervention periods for this cohort. Figure 1 shows our analytic cohort of 1442 UCLA patients with CKD 4-5 including $748(51.9 \%)$ patients who entered the cohort by April 1, 2014, 
and 694 (48.1\%) patients who entered between April 2, 2014, and September 30, 2016. CKD 4-5 patients represented the top $8.4 \%$ of per member per month expenditures within the UCLA attributed patient population. Between April 1, 2013, and September 30, 2016, across 40 practices in Los Angeles, 573,248 patients were attributed to UCLA with 292,170 (51.0\%) in managed contracts. Consistent with national prevalence data, 43,898 (7.7\%) patients had a diagnosis of CKD (based on estimated glomerular filtration rate (eGFR) < $60 \mathrm{~mL} / \mathrm{min} / 1.73 \mathrm{~m}^{2}$ ), and $1507(0.5 \%)$ had CKD 4-5. ${ }^{44}$

At the time of study entry, there were 1084 (75.2\%) CKD stage 4 and 358 (24.8\%) CKD stage 5 patients; 687 (47.6\%) were male. Patients had a median age of 71.9 (IQR 59.7-83.2) and four concurrent conditions (IQR 0-13) (Table 1). Medicare patients were older $(p<0.001)$, were more likely to be non-Hispanic $(p=$ $0.004)$, and had more concurrent conditions $(p<0.001)$ compared

Table 1 CKD 4-5 Patients in UCLA Primary Care Population $(n=$ 1442). Patients with CKD Stages 4 and 5 Represented the Top $8.4 \%$ of per Member per Month Expenditures Within the UCLA Attributed Patient Population

\begin{tabular}{ll}
\hline Characteristics & $\begin{array}{l}\text { Total high- } \\
\text { expense CKD }\end{array}$ \\
\hline Median age in years (IQR) & $71.9(59.7-83.2)$ \\
Male gender, $N(\%)$ & $687(47.6 \%)$ \\
Hispanic ethnicity, $N(\%)$ & $248(17.2 \%)$ \\
Race, $N(\%)$ & $650(45.1 \%)$ \\
Non-Hispanic white or Caucasian & $126(8.7 \%)$ \\
Black or African American & $179(12.4 \%)$ \\
Asian & $7(0.5 \%)$ \\
Native Hawaiian/ Pacific Islander & $232(16.1 \%)$ \\
Other & $1084(75.2 \%)$ \\
CKD stage (study entry), $N(\%)$ & $358(24.8 \%)$ \\
Stage 4 & $4(0-13)$ \\
Stage 5 & $398(27.6 \%)$ \\
Median number of concurrent conditions-CMS & \\
definition ${ }^{\alpha}$ (IQR) & $723(50.1 \%)$ \\
Diabetes, ${ }^{\beta} N(\%)$ & $235(16.3 \%)$ \\
Hypertension, ${ }^{\oplus} N(\%)$ & \\
High-utilizer patient defined by UCLA health & \\
system ${ }^{\Psi} N(\%)$ & $1033(71.6 \%)$ \\
High-risk patient defined by UCLA health & \\
system, $N(\%)$ & \\
\hline
\end{tabular}

${ }^{\alpha}$ CMS includes the following concurrent conditions in defining comorbidities used to calculate the number of concurrent conditions. acquired hypothyroidism, acute myocardial infarction, Alzheimer's disease, Alzheimer's disease/related disorders/or senile dementia, anemia, asthma, atrial fibrillation, benign prostatic hyperplasia, colorectal cancer, endometrial cancer, breast cancer, prostate cancer, cataract, chronic kidney disease, chronic obstructive pulmonary disease, depression, diabetes, glaucoma, heart failure, hip/pelvic fracture, hyperlipidemia, hypertension, ischemic heart disease, osteoporosis, rheumatoid arthritis/osteoarthritis, stroke/transient ischemic attack

${ }^{\beta}$ Patients are defined as having diabetes if they have orders for diabetes medications in the past 5 years, one or more inpatient visits or two or more outpatient visits $w /$ diabetes diagnoses in the past 5 years

${ }^{\epsilon}$ Patients are defined as having hypertension if in the last 24 months, they have a hypertension diagnosis (i.e., members 18-59 years of age without a diagnosis of diabetes whose BP was $>140 / 90 \mathrm{mmHg}$ and members 60-85 years of age without a diagnosis of diabetes whose BP was $>150 / 90 \mathrm{mmHg}$ ) on either 2 outpatient visits or 1 observation visit ${ }^{\Psi} U C L A$ defines high utilizer as those who meet any of the following criteria: (1) 4 or more ED visits in the last 12 months; (2) 2 or more emergent, non-maternity inpatient admissions in the last 12 months

${ }^{\infty}$ UCLA defined high-risk patients as those who meet any of the following criteria: (1) most recent Hbalc $>9 \%$; (2) most recent eGFR $<45 \mathrm{~mL} / \mathrm{min} / 1.73 \mathrm{~m}^{2}$; (3) advanced cancer patient; (4) cirrhosis patient with MELD > 16; (5) high opioid equivalent use with non-Medicare patients (Appendix - Section II, exhibit A). Original entrant patients were older $(p<0.001)$, were white or black $(p<0.001)$, were high utilizers $(p<0.001)$, and had more concurrent conditions $(p<0.001)$ than new entrant patients (Appendix - Section II, exhibit B).

Table 2 respectively displays results of interrupted time series regression analyses for four study outcomes: hospitalization, bed days, ED visits, and 30-day all-cause readmission. The rate of hospitalizations per 1000 patient-years declined at an estimated monthly rate of $2.0 \%$ during the pre-intervention period (95\% CI: $-3.2 \%,-0.8 \%)$. After intervention implementation, the estimated rate of decline was $5.4 \%$ (95\% CI: $7.4 \%,-3.4 \%), 3.5$ percentage points faster than the preintervention decline of $2.0 \%$ per month $(95 \%$ CI: $-5.8 \%$, $1.1 \% ; p=0.004)$.

The number of inpatient bed days per 1000 patient-years declined during the pre-intervention period at an estimated rate of $4.8 \%$ per month $(95 \% \mathrm{CI}:-7.0 \%,-2.5 \%)$. After intervention implementation, the estimated rate of decline was $8.6 \%$ per month (95\% CI: $-12.3 \%,-4.7 \%)$. While not statistically significant, this was 4.0 percentage points faster than the pre-intervention rate of decline (95\% CI: $-8.5 \%, 0.7 \% ; p=0.10)$.

Monthly ED visits during the pre-intervention period declined at an estimated rate of $0.9 \%$ per month $(95 \%$ CI: $2.0 \%,-0.1 \%$ ). After intervention implementation, the estimated rate of decline for the ED visit rate was $2.7 \%$ per month (95\% CI: $-4.3 \%,-0.9 \%)$. While this was not statistically significant, it was 1.7 percentage points faster than the preintervention rate of decline (95\% CI: $-3.7 \%, 0.3 \% ; p=0.09$ ). There was no difference in 30-day all-cause readmission trends (95\% CI: $-33.5,11.8 \% ; p=0.26)$.

After stratifying the outcomes by payer, we found Medicare patients' average ED visit rate of decline was $3.0 \%$ per month (95\% CI: $-4.8 \%,-1.2 \%)$, which was significantly faster (2.6 percentage points, $95 \%$ CI: $0.4 \%, 4.7 \% ; p=0.02$ ) than the preintervention decline of $0.4 \%$ per month $(95 \% \mathrm{CI}:-1.6 \%$, $0.8 \%$ ). The rate of decline among non-Medicare patients across the same pre-intervention and post-intervention periods was not significant ( $p=0.28$, not shown). Overall, the intervention effect on monthly ED visits was significantly greater for Medicare patients compared to those with other types of insurance $(p=0.03)$. In contrast, we found no significant differences in outcome trends after stratifying the cohort by either original and new entry patients or by CKD stages $4-5$ at baseline (not shown). Our results remained unchanged in sensitivity analyses in which we included the intervention implementation period as a further study period.

\section{DISCUSSION}

This evaluation is one of the first empirical analyses of a large, health system-wide population health approach for acute clinical decline of CKD 4-5 patients. UCLA implemented the PHV-CKD approach to provide timely services while reducing healthcare 
Table 2 Monthly Percent Change in Utilization Outcomes Based on Interrupted Time Series Design $(n=1442)$

\begin{tabular}{|c|c|c|c|c|}
\hline \multirow[b]{2}{*}{$\begin{array}{l}\text { Utilization outcomes per } 1000 \\
\text { patient-years }{ }^{\alpha}\end{array}$} & \multirow{2}{*}{$\begin{array}{l}\text { Pre-intervention period }(4 / 1 / \\
15-9 / 30 / 16) \\
\\
\text { Percent change per month } \\
(95 \% \text { CI })\end{array}$} & \multirow{2}{*}{$\begin{array}{l}\text { Post-intervention period }(1 / 1 / 17- \\
12 / 31 / 17) \\
\text { Percent change per month }(95 \% \\
\text { CI) }\end{array}$} & \multicolumn{2}{|c|}{$\begin{array}{l}\text { Difference (post-pre-intervention } \\
\text { periods) }\end{array}$} \\
\hline & & & Difference (95\% CI) & $p$ value $^{\beta}$ \\
\hline Hospitalizations & $-2.0 \%(-3.2 \%,-0.8 \%)$ & $-5.4 \%(-7.4 \%,-3.4 \%)$ & $\begin{array}{l}-3.5 \%(-5.8 \%,- \\
1.1 \%)\end{array}$ & $0.004 * * *$ \\
\hline All inpatient bed days & $-4.8 \%(-7.0 \%,-2.5 \%)$ & $-8.6 \%(-12.3 \%,-4.7 \%)$ & $\begin{array}{l}-4.0 \%(-8.5 \% \\
0.7 \%)\end{array}$ & $0.10^{+}$ \\
\hline ED visits & $-0.9 \%(-2.0 \%, 0.1 \%)$ & $-2.7 \%(-4.3 \%,-0.9 \%)$ & $\begin{array}{l}-1.7 \%(-3.7 \% \\
0.3 \%)\end{array}$ & $0.09^{+}$ \\
\hline 30-day all-cause readmission & $4.2 \%(-8.3,18.4 \%)$ & $-10.2 \%(-28.3 \%, 12.6 \%)$ & $\begin{array}{l}-13.8 \%(-33.5 \% \\
11.8 \%)\end{array}$ & 0.26 \\
\hline
\end{tabular}

We analyzed the utilization data from the standpoint of an interrupted time series design, using a segmented regression modeling strategy. The model was used to estimate separate log-linear time trends in the period before, and the period after, intervention rollout. This was implemented by including in the negative binomial marginal model terms for period (pre-versus post-intervention), time (modeled log-linearly in calendar months), and their interaction. Model contrasts were used to estimate the monthly rate of change in incidence rates before and after the intervention, and the interaction term was used to test for a change in trends. In each of the regression analyses, the unit of analysis was patient-month, and the response variable was the count of the relevant utilization events. Analyses were clustered at the patient level to correct inferences for the presence of repeated measures ${ }^{\beta}$ All utilization outcomes are adjusted for patient age, gender, race, ethnicity, high risk status, high utilizer status, and comorbid condition count ${ }^{\beta} p$ values indicate the significance of the difference in utilization between the pre- and the post-intervention periods as ${ }^{+} p<0.10 ; * * p<0.05$; $* * * p<0.01$. CI, confidence interval

utilization. The analysis, based on an interrupted time series design, demonstrated that hospitalization rates reduced more rapidly after implementation across the study population. Monthly ED visits, but not monthly bed days or readmissions, ${ }^{45}, 46$ also reduced at a significantly faster rate among Medicare-insured patients known to have high medical complexity (Table 1). We anticipated that the reduction in hospitalizations and ED visits would occur first, while we believed further inpatient-based interventions may ultimately associate with reduced length of stay and hospital readmissions. The latter outcomes would require improving clinical discharge planning (e.g., needing physical therapy evaluation or new medical equipment), inpatient care management, and social work services, which we did not specifically measure as part of our evaluation.

Previous population health approaches in primary and specialty care have demonstrated only modest, if any, reductions in inpatient utilization and expenses for patient populations requiring multispecialty care. ${ }^{27,47-55}$ Limitations of previous programs include (1) leadership residing within siloed specialties that limit system-wide implementation, (2) patients not completing specialty clinic visits, ${ }^{18,26,27,56}$ (3) local data constraints that limit responsiveness to patient needs, ${ }^{21,50,55-60}$ (4) unclear identification of patients most likely to benefit from interventions, ${ }^{61}$ (5) nebulous primary and specialty care clinical alignment, ${ }^{18,} 26,53$, ${ }^{62-65}$ and (6) narrowly resourced ambulatory care services. ${ }^{66}$

The PHV-CKD intervention accounted for various ways that patients present with clinical decline or need to rapidly link them to required care. System-wide strategies included creating a robust care coordinator network across primary and specialty care enabled by EHR tools and increased ambulatory access. There has been one similar randomized trial among CKD 4-5 patients focused in a more controlled setting of three specialty clinics and found pronounced reductions in hospitalizations and ED visits. ${ }^{25}$ As other health systems embarking in similar efforts, they can gain different insights from this system-wide model across a broad primary care population with CKD 4-5.
A multi-source data infrastructure facilitated care coordinators in identifying patients who met criteria for clinical decline or need and allocating resources to meet patients' needs (e.g., extending service hours). In contrast to previously cited costneutral programs, ${ }^{7,} 61,67$ the UCLA PHV-CKD intervention used empirical analyses to identify a catchment of patients who may most benefit to optimize efficiency.

The intervention aligned clinicians into a system-wide effort using care coordination pathways rather than placing care coordinators within multiple siloed specialties that function independently ${ }^{18,}{ }^{26}$ Without a coordinated, team-based multispecialty approach, care teams risk delivering inconsistent, duplicative, and expensive care. ${ }^{27-31,68}$ While previous care management programs have relied on $\mathrm{PC}-\mathrm{CCs},{ }^{33,69,70}$ leaders found that patients benefited from care coordination that could also span specialty and community practices. The PHV intervention, therefore, allowed system-wide care coordinators to proactively identify patients with clinical decline or need to prevent hospitalizations. This approach differs from prior studies of CKD care coordination that have focused on other quality of care outcomes more than hospitalization reduction. ${ }^{26}$

Study limitations include a changing patient population over time, secular trends, measuring other outcomes of value, and generalizability. UCLA underwent an expansion over the prior 5 years and acquired additional primary care practices. ${ }^{23}$ In response, we added analyses comparing original versus new entry patients which found no significant differences in outcomes. There is potential that other ACO efforts such as primary care-based care coordination and behavioral health integration could have influenced measured outcomes. However, for the first time, with this new intervention, the health system focused on improving outcomes and efficiency system-wide for CKD 4-5 patients. While this is a single-site study which limits generalizability, the model rests on universal concepts including development of population approaches to meet patient needs. We focused 
on four clinically relevant, valid outcomes though we recognize potentially relevant outcomes that we were unable to measure. Our analyses do not include direct cost data or reimbursement data because these data were inaccessible and incomplete. Patient satisfaction data is not included because it was anonymously collected and could not be feasibly linked to study data. We do not specifically report unnecessary inpatient utilization and ED visits as there is currently no validated measure of unnecessary inpatient utilization. Finally, the analysis lacked a comparison control group, as the intervention was implemented system-wide and logistics limited using time-shifted comparison evaluation.

Next steps include expanding secondary prevention to CKD stage 1-3 patients. We also plan to adapt this model to other high-risk sub-populations and medical centers.

\section{CONCLUSION}

By creating primary-specialty care pathways with increased ambulatory infrastructure, healthcare systems can enhance care coordination to address patient needs while reducing hospitalizations. These findings also suggest opportunities for policies to incentivize redesigning care for stage 4-5 CKD populations. ${ }^{8,9}$

Acknowledgments: The authors would like to thank Varsenik Papazyann-Gutierrez, Neil Wenger, Connie Lee, Lily Roh, David Elashoff, and Robin Clarke for their participation with the study.

Corresponding Author: R. Gupta, MD, MSHPM; Los Angeles, USA (e-mail: R44gupta@gmail.com).

\section{Compliance with Ethical Standards:}

Conflict of Interest: Dr. Gupta is a Co-Director at Costs of Care, Inc. No other disclosures to report.

\section{REFERENCES}

1. Civic Impulse. (2017). H.R. 2 - 114th Congress: Medicare Access and CHIP Reauthorization Act of 2015. Accessed May 9, 2020: https://www. govtrack.us/congress/bills/114/hr2.

2. Alternative Payment Model Framework and Progress Tracking (APM FPT) Working Group. (2016). Alternative Payment Model (APM) Framework: Final White Paper. Retrieved from https://hcp-lan.org/workproducts/apmwhitepaper.pdf

3. Gupta R. Collateral damage occurs when doctors and patients wear 'Covid-19 blinders.' STAT. Published May 4, 2020. Accessed May 9, 2020: https://www.statnews.com/2020/05/04/collateral-damage-occurswhen-doctors-and-patients-wear-covid-19-blinders/

4. Vanholder R, Annemans L, Brown E, Gansevoort R, Gout-Zwart JJ, Lameire N, Morton RL, Postma MJ, Tonelli M, Van Biesen W, Zoccali C. Reducing the costs of chronic kidney disease while delivering quality health care: a call to action. Nature Reviews Nephrology. 2017;13:393409.

5. S. L. Hayes, C. A. Salzberg, D. McCarthy, D. C. Radley, M. K. Abrams, T. Shah, and G. F. Anderson, High-Need, High-Cost Patients: Who Are They and How Do They Use Health Care-A Population-Based Comparison of Demographics, Health Care Use, and Expenditures, The Commonwealth Fund, August 2016.

6. Cohen $\mathbf{S}$, Uberoi $\mathbf{N}$. Differentials in the Concentration in the Level of Health Expenditures Across Population Subgroups in the US, 2010. Rocville, MD: Agency for Healthcare Research and Quality; 2013.
7. Hong C, Siegel A, Ferris T. Caring for High-Need, High-Cost Patients: What Makes for a Successful Care Management Program? New York, NY: Commonwealth Fund: 2014.

8. Trump, Donald J. Executive Orders: Executive Order on Advancing American Kidney Health. Published July 20, 2019. Accessed May 9, 2020: https://www.whitehouse.gov/presidential-actions/executive-order-advancing-american-kidney-health/

9. CMS.gov. HHS To Transform Care Delivery for Patients with Chronic Kidney Disease. Centers for Medicare and Medicaid Services. July 10, 2019: https://www.cms.gov/newsroom/press-releases/hhs-transformcare-delivery-patients-chronic-kidney-disease.

10. Golestaneh, L, Alvarez PJ, Fund SE, McGaughey KJ, Romero A, Brennen MS, Onuigbo M. All-cause costs increase exponentially with increased chronic kidney disease stage. Am J Manag Care. 2017;23(10 Suppl):S163-S172.

11. Damien $\mathbf{P}$, Lanham HJ, Parthasarathy M, Shah NL. Assessing key cost drivers associated with caring for chronic kidney disease patients. BMC Health Services Research. 2016; 16:690-700.

12. U.S. Renal Data System (2018). USRDS 2018 Annual Data Report Highlights: New Report Captures the High Burden, High Cost and Low Awareness of Kidney Disease in the United States. National Institutes of Health, National Institute of Diabetes and Digestive and Kidney Diseases, Bethesda, MD. Accessed May 9, 2020: https://www.usrds.org/ adrhighlights.aspx.

13. Komenda P, Tangri N, Klajncar E, et al. Patterns of emergency department utilization by patients on chronic dialysis: a populationbased study. PLoS One. 2018;13(4):e0195323.

14. Wang, V., Vilme, H., Maciejewski, M. L. \& Boulware, L. E. The economic burden of chronic kidney disease and end-stage renal disease. Semin. Nephrol. 36, 319-330 (2016).

15. Collins, A. J., Foley, R. N., Gilbertson, D. T. \& Chen, S. C. United States Renal Data System public health surveillance of chronic kidney disease and end-stage renal disease. Kidney Int. 2015;(5):2-7.

16. Holley JL. Advance Care Planning in CKD/ESRD: An Evolving Process. CJASN. 2012;7(6):1033-1038.

17. Collins AJ, Foley RN, Herzog C, et al. US Renal Data System 2010 annual data report. Am J Kidney Dis. 2011;57(1)(suppl 1):e1-e526.

18. Fishbane S, Agoritsas S, Bellucci A, et al. Augmented Nurse Care Management in CKD Stages 4 and 5: A Randomized Trial. AJKD. 2017;70(4):498-505. https://www.ajkd.org/article/S0272-6386(17)30547-4/abstract

19. Wong SP, Hebert PL, Laundry RJ, et al. Decisions about renal replacement therapy in patients with advanced kidney disease in the US Department of Veterans Affairs, 2000-2011. Clin J Am Soc Nephrol. 2016 Sep 22. pii: CJN.03760416.

20. Saran R, Li Y, Robinson B, et al. US Renal Data System 2015 annual data report: epidemiology of kidney disease in the United States. Am J Kidney Dis. 2016;67(3)(suppl 1):S1-S434.

21. Collins AJ, Foley RN, Gilbertson DT, Chen SC. The state of chronic kidney disease, ESRD, and morbidity and mortality in the first year of dialysis. Clin J Am Soc Nephrol. 2009;4(suppl 1):S5-S11.

22. End-Stage Renal Disease in the United States. Chapter 4: Hospitalizations. United States Renal Data System. 2017; 2: 321-335.

23. Phone conversations with Lily Roh, Director of Population Health at UCLA on January 18, 2019 and James Wilson, Medical Director of UCLA Stone Center and Surgical Consultative Nephrology Service on March 29, 2019.

24. U.S. Renal Data System (2018). USRDS 2018 Annual Data Report: Atlas of Chronic Kidney Disease and End-Stage Renal Disease in the United States. National Institutes of Health, National Institute of Diabetes and Digestive and Kidney Diseases, Bethesda, MD. Accessed March 10, 2019: https://www.usrds.org/2018/download/v2_c04_Hospitalization_18_ usrds.pdf. Volume 2, p. 387-410.

25. U.S. Renal Data System (2018). USRDS 2018 Annual Data Report: Atlas of Chronic Kidney Disease and End-Stage Renal Disease in the United States. National Institutes of Health, National Institute of Diabetes and Digestive and Kidney Diseases, Bethesda, MD. Accessed May 9, 2020: https://www.usrds.org/2018/download/v1_c02_IdentCare_18_usrds. pdf. Volume 1, p. 29-44.

26. Nicoll R, Robertson L, Gemmell E, et al. Models of care for chronic kidney disease: A systematic review. Nephrology. 2018;23:389-396. https://www.ncbi.nlm.nih.gov/pubmed/29160599

27. Smith ZG, McNicoll L, Clark TL, Cohen AJ, Ross AL, Monteiro JF, Dworkin LD. Medical Neighborhood Model for the Care of Chronic Kidney Disease Patients. Am J Nephrol. 2016; 44(4):308-315.

28. Lisa M. Kern et al. Fragmented Ambulatory Care and Subsequent Healthcare Utilization Among Medicare Beneficiaries. Am J Manag Care. 2018; 24(9):294-300. 
29. Meltzer DO, Ruhnke GW. Redesigning Care for Patients at Increased Hospitalization Risk: the Comprehensive Care Physician Model. Health Affairs. 2014;33(5):770-777.

30. Becker GS, Murphy KM. The division of labor, coordination costs, and knowledge. Q J Econ. 1992; 107(4):1137-60.

31. Murphy J, Chang H, Montgomery JE, Rogers WH, Safran DG. The quality of physician-patient relationships: patients' experiences 19961999. J Fam Pract. 2001; 50(2):123-9.

32. O'Malley AS, Reschovsky JD. Referral and consultation communication between primary care and specialist physicians: finding common ground. Arch Intern Med 2011;171: 56-65.

33. Clarke R, Bharmal N, Di Capua $\mathbf{P}$, et al. Innovative approach to patientcentered care coordination in primary care practices. Am J Managed Care. 2015; 21(9): 623-630.

34. Silver SA \& Chertow GM. Cost of Acute Kidney Injury in Hospitalized Patients. J Hosp Med. 2017; 12:70-76.

35. Fitch K, Engel T, Lau J. The Cost Burden of Worsening Heart Failure in the Medicare Fee for Service Population: an Actuarial Analysis. Milliman Report. Accessed May 9, 2020: http://us.milliman.com/uploadedFiles/ insight/2017/cost-bruden-worsening-heart-failure.pdf. Published 2017.

36. Clarke R, Jeffrey J, Grossman M, Trouse T, Gitlin M, Skootsky S Delivering On Accountable Care: Lessons From A Behavioral Health Program To Improve Access And Outcomes. Hlth Aff. 2016;35(8): 14871493.

37. Gupta R, Roh L, Lee C, Reuben D, Naeim A, Wilson J, Skootksy S Reducing Total Costs of Care in Creating Value through Population Health: Focusing on High Cost Conditions. Acad Med. 2019 Sep;94(9):1337-1342. doi: https://doi.org/10.1097/ACM. 0000000000002739

38. Clarke R, Hackbarth AS, Saigal C, et al. Building the Infrastructure for Value at UCLA: Engaging Clinicians and Developing Patient-Centric Measurement. Acad Med 2015 Oct;90(10):1368-72.

39. Benin AL, Fenick A, Herrin J, Vitkauskas G, Chen J, Brandt C. How good are the data? Feasible approach to validation of metrics of quality derived from an outpatient electronic health record. American Journal of Medical Quality. 2011 Nov;26(6):441-51

40. Miller, WR \& Rollnick, S. (2009). Ten things that Motivational Interviewing is not. Behavioural and Cognitive Psychotherapy, 37, $129 \mathrm{~F} 140$.

41. Wanger AK, Somerai SB, Zhang F, et al. Segmented regression analysis of interrupted time series studies in medication use research. J Clin Pharm and Therap. 2002;27(4):299-309.

42. Taljaard M, McKenzie JE, Ramsay et al. The use of segmented regression in analyzing interrupted time series studies: an example in pre-hospital ambulance care. Implement Sci. 2014;9:77.

43. Ballinger GA. Using Generalized Estimating Equations for Longitudinal Data Analysis. Organizational Research Methods, Vol. 7 No. 2, April 2004 127-150.

44. Murphy D, McCulloch CE, Lin F, et al. Trends in prevalence of chronic kidney disease in the United States. Ann Intern Med. 2016;165(7):473481.

45. Leppin, A.L., Gionfriddo, M.R., Kessler, M. et al. Preventing 30- day hospital readmissions: a systematic review and meta-analysis of randomized trials. JAMA Intern Med. 2014; 174: 1095-1107.

46. Mathew A, Strippoli GFM, Ruospo M, et al. Reducing hospital readmissions in patients with end-stage kidney disease. Kidney International. 2015;88(6): 1250-1260.

47. The U.S. Department of Health and Human Services. Defining the PCMH AHRQ. Accessed May 9, 2020: https://pcmh.ahrq.gov/page/definingpcmh.

48. Taylor EF, Lake T, Nysenbaum J, et al. Coordinating Care in the Medical Neighborhood: Critical Components and Available Mechanisms: White Paper. AHRQ. Published June 2011. Accessed May 9, 2020: https://pcmh.ahrq.gov/sites/default/files /attachments / Coordinating\%20Care\%20in\%20the\%20Medical\%20Neighborhood.pdf.

49. Edwards ST, Bitton A, Hong J, Landon BE. Patient-Centered Medical Home Initiatives Expanded in 2009-13: Providers, Patients, and Payment Incentives Increased. Health Affairs. 2013; 33(1): 1823-31.
50. ACP. The patient-centered medical home neighbor: the interface of the patient-centered medical home with specialty/subspecialty practices. Philadelphia: ACP, 2010. Accessed May 9, 2020: http://www.acponline. org/advocacy/where we stand/policy/pcmh_neighbors.pdf.

51. Duke Institute for Health Innovation. Building a Virtual Medical Neighborhood for Chronic Kidney Disease in Duke Primary Care. Published 2018. Accessed May 9, 2020: https://dihi.org/projects/building-virtualmedical-neighborhood-chronic-kidney-disease-duke-primary-care.

52. Powers BW, Chaguturu SK, Ferris TG. Optimizing high-risk care management. JAMA. 2015 Feb 24;313(8):795-796

53. Jackson GL, Powers BJ, Chatterjee R, et al. The patient-centered medical home: a systematic review. Ann Intern Med 2013;158: 169-78.

54. Congressional Budget Office. Lessons from Medicare's demonstration projects on disease management, care coordination, and value-based payment [Internet]. Washington (DC): CBO. Accessed May 9, 2020 http://www.cbo.gov/sites/default/files/cbofiles/attachments/01-1812-MedicareDemoBrief.pdf. Published Jan 2012.

55. Peikes D, Dale S, Ghosh A, et al. The Comprehensive Primary Care Initiative: Effects on Spending, Quality, Patients, and Physicians. Hlth Aff. 2018; 37 (6): 890-899.

56. Huang $\mathbf{X}$, Rosenthal MB. Transforming Specialty Practice- The Patientcentered Medical Neighborhood. N Engl J Med. 2014; 370 (75): 13761379 .

57. Beckman AL, Chmar L, Broome T, Kocher B, Mostashari F. Payment Designed or People Introducing the Primary Care Outcomes Model. NEJM Catalyst. Published October 23, 2018. Accessed Dec 1, 2018 https://catalyst.nejm.org/introducing-primary-care-outcomes-model/

58. Blumenthal D, Abrams MK. Tailoring Complex Care Management for High-Need, High-Cost Patients. JAMA 2016;316(16):1657-1658.

59. Lynn J, Straube BM, Bell KM, Jencks SF, Kambic RT. Using population segmentation to provide better health care for all: the "Bridges to Health" model. Milbank Q. 2007;85(2):185-208; discussion 209-112.

60. Yan J, Linn KA, Powers BW, Zhu J, Jain SH, Kowalski JL, Navathe AS. Applying Machine Learning Algorithms to Segment High-Cost Patient Populations. J Gen Intern Med. 2018;1(33):1-7. https://doi.org/10. 1007/s11606-018-4760-8

61. Bodenheimer T, Berry-Millett R. Care Management of Patients with Complex Health Care Needs, Research Synthesis Report No. 19 (Princeton, N.J.: Robert Wood Johnson Foundation, Dec. 2009).

62. Kirschner N , Barr MS . Specialists subspecialists and the patientcentered medical home . Chest . 2010 ; 137 ( 1 ): 200 - 204

63. Starfield B. Primary Care, Specialist Care, and Chronic Care. Can They Interlock? CHEST. 2010; 137(1): 8-10.

64. Starfield B , Chang HY, Lemke KW, Weiner JP . Ambulatory specialis use by nonhospitalized patients in US health plans: correlates and consequences. J Ambul Care Manage. 2009;32(3):216 - 225.

65. Bitton A. Who is on the Home Team? Redefining the Relationship between Primary and Specialty Care in the Patient-Centered Medical Home. Medical Care. 2011;49(1):1-3

66. Rosano A, Loha CA, Falvo R, et al. The relationship between avoidable hospitalization and accessibility to primary care: a systematic review. European Journal of Public Health. 2013;23(3):356-360.

67. Coleman EA, Parry C, Chalmers S, Min SJ. The care transitions intervention. Arch Intern Med. 2006; 166(17):1822-8.

68. Pham HH, Schrag D, O'Malley AS, et al. Care Patterns in Medicare and Their Implications for Pay for Performance. N Engl J Med. 2007; 356: 1130-1139.

69. Gill JM, Mainous AG 3rd. The role of provider continuity in preventing hospitalizations. Arch Fam Med. 1998;7(4):352-7.

70. Wasson JH, Sauvigne AE, Mogielnicki RP, Frey WG, Soc CH, Gaudette C, et al. Continuity of Outpatient medical care in elderly men. A randomized trial. JAMA. 1984; 252(17):2413-7.

Publisher's Note: Springer Nature remains neutral with regard to jurisdictional claims in published maps and institutional affiliations. 